METZLER GOETHE LEXIKON 


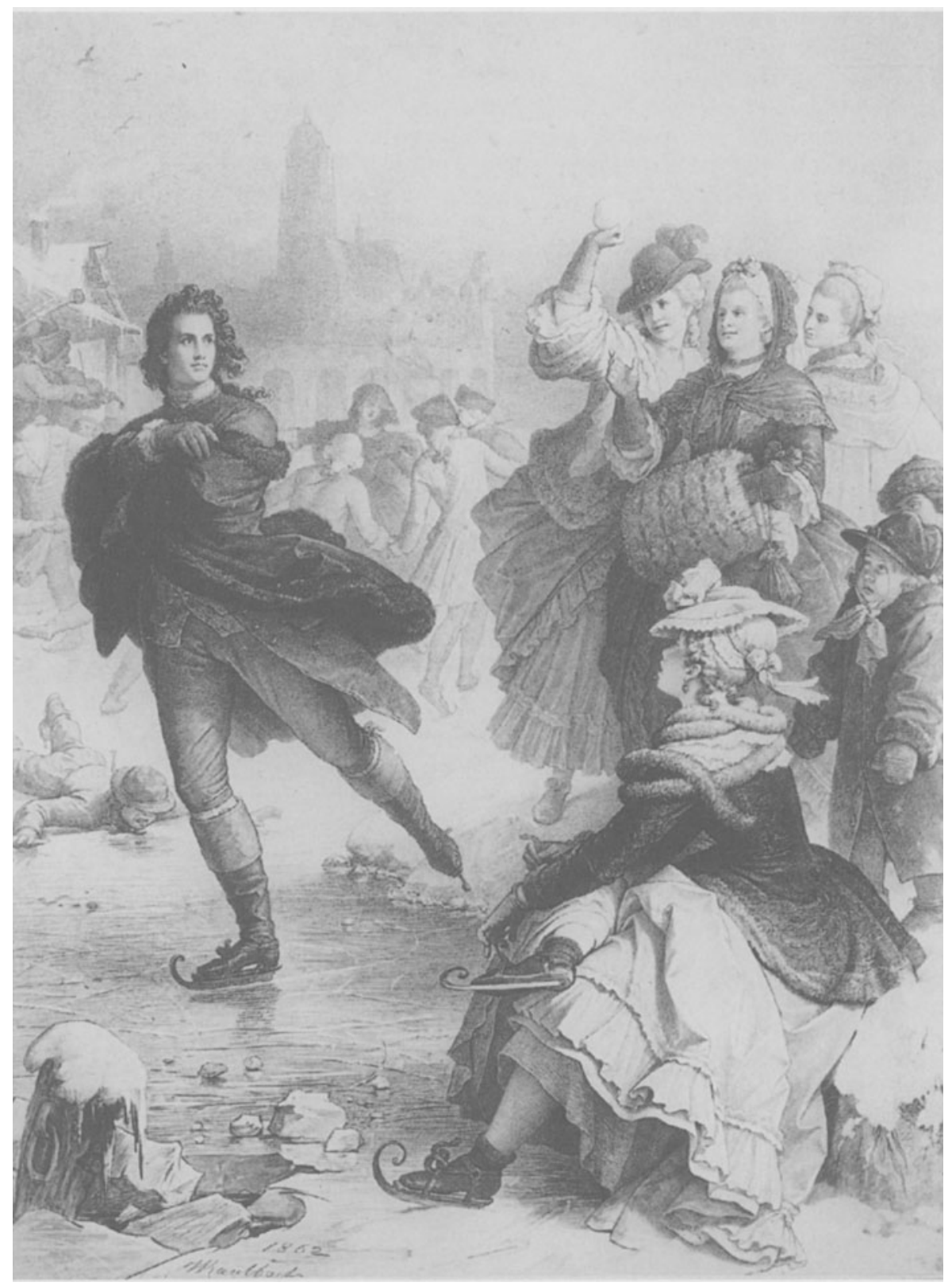

-Der junge Goethe auf dem Eise. Karton von Wilhelm von Kaulbach 
Ein sebr harter Winter batte den Main völlig mit Eis bedeckt und in einen festen Boden verwandelt. Der lebhafteste, notwendigste und lustig gesellige Verkebr regte sich auf dem Eise. Grenzenlose Schlittschubbahnen, glattgefrorne weite Stellen wimmelten von bewegter Versammlung. Ich feblte nicht vom früben Morgen an und war also, wie späterbin meine Mutter, dem Schauspiel zuzusehen, angefabren kam, als leichtgekleidet wirklich durchgefroren. Sie saß im Wagen mit ibrem roten Sammetpelze, der, auf der Brust mit starken goldenen Schnüren und Quasten zusammengehalten, ganz stattlich aussah. "Geben Sie mir, liebe Mutter, Ibren Pelz!‘ rief ich aus dem Stehgreife, obne mich weiter besonnen zu baben, "mich friert grimmig. Auch sie bedachte nichts weiter; im Augenblick batte ich den Pelz an, der purpurfarb bis an die Waden reichend, mit Zobel verbrämt und mit Gold geschmückt, zu der braunen Pelzmütze, die ich trug, gar nicht übel kleidete. So fubr ich sorglos auf und ab, auch war das Gedränge so groß, daß man die seltene Erscheinung nicht einmal sonderlich bemerkte, obschon einigermaßen: denn man rechnete sie mir unter meinen Anomalien im Ernst und im Scherze wobl einmal wieder vor.

(DuW, IV.16) 


\title{
METZLER GOETHE LEXIKON
}

\author{
Mit 150 Abbildungen
}

Herausgegeben von Benedikt Jeßing,

Bernd Lutz und Inge Wild

Redaktion: Sabine Matthes

Verlag J.B. Metzler

Stuttgart · Weimar 


\section{Inhalt}

Vorwort VII

Verzeichnis der Autorinnen und Autoren VIII

Siglenliste VIII

Artikel von A bis Z 1

Chronik: Goethes Leben und Werk $\mathbf{5 6 5}$

Weiterführende Bibliographie $\mathbf{5 7 7}$

Bildquellen $\mathbf{5 8 0}$

Die Deutsche Bibliothek - CIP-Einheitsaufnahme

Metzler-Goethe-Lexikon : alles über Personen, Werke, Orte, Sachen,

Begriffe, Alltag und Kurioses ; mit 2200 Artikeln / hrsg. von

Benedikt Jeßing ... - Stuttgart ; Weimar : Metzler, 1999

ISBN 978-3-476-01589-1

ISBN 978-3-476-03732-9 (eBook)

DOI 10.1007/978-3-476-03732-9

Dieses Werk einschließlich aller seiner Teile ist urheberrechtlich geschützt. Jede Verwertung außerhalb der engen Grenzen des Urheberrechtsgesetzes ist ohne Zustimmung des Verlages unzulässig und strafbar. Das gilt insbesondere für Vervielfältigungen, Übersetzungen, Mikroverfilmungen und die Einspeicherung und Verarbeitung in elektronischen Systemen.

C 1999 Springer-Verlag GmbH Deutschland

Ursprünglich erschienen bei J.B. Metzlersche Verlagsbuchhandlung

und Carl Ernst Poeschel Verlag GmbH in Stuttgart 1999 


\section{"In tausend Formen magst du dich verstecken«: Von der Belebung der Welt durch Poesie und Liebe}

Die Arbeit, das Schreiben an diesem Goethe-Lexikon hat allen beteiligten Autorinnen und Autoren - Kulturwissenschaftler, Historiker, Musikwissenschaftler, Dramaturgen, Literaturwissenschaftler, Museumspädagogen - viel Freude bereitet. Die Aufgabe bestand darin, ein Großwerk der europäischen Literatur aus Anlaß des 250. Geburtstags seines Verfassers kompakt und kompetent einem an der Sache der Literatur interessierten Publikum neu zugänglich zu machen.

Das Lexikon versucht, unsere gegenwärtige, moderne Lesart Goethes, seines Lebens, seiner Werke, seiner Zeit, wiederzugeben; die Tatsache also, daß die interpretierenden Lektüren von Lyrik, Dramatik und Prosa sowie der Briefe, Amtlichen Schriften usf. immer auch zu einem Teil die subjektive Lesart der jeweiligen Autoren repräsentieren - hier werden nicht nur Fakten geboten, sondern auch mögliche Verständnisweisen der heutigen Zeit.

Nachzuzeichnen war der Lebensweg eines frühzeitig - nach dem Erscheinen des Briefromans Die Leiden des jungen Werthers und der Uraufführung des Götz von Berlichingen - mit dem Prädikat des "Genialen* versehenen Autors, auf dem das Auge der deutschen und - erstmals im geschichtlichen Verlauf der deutschsprachigen Literatur - der europäischen Öffentlichkeit ruhte und daher einzigartig dicht dokumentiert und kommentiert ist. Um dieses universalistische Konzept - G. Auffassung seiner Persönlichkeit selbst - als kulturhistorisch interessierter Leser nachvollziehen zu können, enthält das Goethe-Lexikon zahlreiche Schlüsselartikel: Die "Chronik: Goethes Leben und Werk" (am Schluß des Goethe-Lexikons), "Amtliche Tätigkeiten", "Bildende Künste", "Bühnenbearbeitungen ", "Carl August, Herzog von Sachsen-Weimar-Eisenach ", "Deutschunterricht «", matiker «, "Einkommen «, "Erlösung «, "Frauen um G.", "Geselligkeit «,"Haushaltsführung «,"Hoftheater «,"Lied «, "Lyriker «, "Macht«, "Maschinenwesen «, "Metternich", "Moderne«, "Musikalität«, Musiktheater «, "Napoleon", "Naturwissenschaften ", "Prosaschriftsteller ", "Selbstmord a, "Tod «, "Weimar «, "West-östlicher Divan", "Zukunft“. Dies nur zur vorläufigen Auswahl und zur Erweckung von Entdeckerlust.

Die Werktitel und Textzitate folgen der Hamburger Ausgabe (Hg. Erich Trunz), der heute am weitesten verbreiteten Goethe-Ausgabe, wenn nicht anders angegeben. Brief- und Gesprächszitate werden den Kalenderdaten nach genannt und sind somit auch in anderen Werkausgaben auffindbar. Von den mit einem Pfeil versehenen Verweisen auf andere Artikel wurde sparsam Gebrauch gemacht: Sowohl bei Werknennungen (kursiv) als auch bei Namensnennungen ist in der Regel auf einen Verweispfeil verzichtet worden, es sei denn, es ergebe sich ein besonders signifikanter, weiterführender Informationsbezug.

Die Autorinnen und Autoren dieses Goethe-Lexikons haben keineswegs bei "null «, bei den "Quellen" beginnen müssen. Sie stützen sich, zu vielfältigstem Dank verpflichtet, auf die reich vorhandene GoetheLiteratur, die großen Ausgaben, deren Kommentare und das neue, mehrbändige Goethe-Handbuch, das im selben Verlag erschienen ist. Die für dieses GoetheLexikon grundlegende und zugleich weiterführende Literatur wurde in der abschließenden Bibliographie verzeichnet.

Wenn es den Autorinnen und Autoren dieses Goethe-Lexikons gelungen sein sollte, in einem Jahr der sich überschlagenden "Neuigkeiten zu Goethe" den beeindruckenden Kosmos der Goetheschen Welterfahrung auf ein verträgliches Maß beschränkt zu haben, das nachvollziehbar ist, damit begeistert und zur Lektüre Goethes selbst verpflichtet, gleichzeitig aber die Spannweite eines Denkens und Schaffens spürbar macht, das im modernen Europa seiner Zeit - andere Große waren früher da oder kamen später - seinesgleichen zu suchen hatte, würden Herausgeber, Redakteurin, Autorinnen und Autoren ihre darstellerische Absicht, die letzten Endes eine historische, eine bescheiden erzählende ist, gelungen sehen.

Dennoch bleibt ein wenig Lampenfieber, über das der Theaterdirektor, zumindest rhetorisch betroffen und sich vor seinem zahlenden Publikum verbeugend, in der Vorrede zu Faust. Der Tragödie erster Teil, v. 41-48, berichtet:
Sie sitzen schon, mit boben Augenbraunen, Gelassen da und möchten gern erstaunen. Ich weiß, wie man den Geist des Volks versöbnt; Doch so verlegen bin ich nie gewesen: Zwar sind sie an das Beste nicht gewöhnt, Allein sie haben schrecklich viel gelesen. Wie machen wir's, daß alles frisch und neu Und mit Bedeutung auch gefällig sei?

Bochum, Karlsruhe, Stuttgart, im März 1999.

Benedikt Jeßing, Bernd Lutz, Sabine Matthes, Inge Wild. 


\section{Verzeichnis der Autorinnen und Autoren}

$\begin{array}{llll}\text { AD: } & \text { Amadeus Deutsch } & \text { HGM: } & \text { Herbert Greiner-Mai } \\ \text { AE: } & \text { Annette Eberhard } & \text { HM: } & \text { Heike Maier } \\ \text { AK: } & \text { Anja Krutinat } & \text { IA: } & \text { Ingo Anhenn } \\ \text { AR: } & \text { Alexander Reck } & \text { IW: } & \text { Inge Wild } \\ \text { AV: } & \text { Albert Vinzenz } & \text { JAS: } & \text { Jan-Arne Sohns } \\ \text { AvG: } & \text { Amelie von Graevenitz } & \text { JK: } & \text { Johannes Kunsemüller } \\ \text { BB: } & \text { Brigitte Bader } & \text { KHF: } & \text { Karl-Heinz Fingerhut } \\ \text { BJ: } & \text { Benedikt Jeßing } & \text { M-LK: } & \text { Marie-Luise Kahler } \\ \text { BL: } & \text { Bernd Lutz } & \text { NH: } & \text { Niklaus Helbling } \\ \text { BS: } & \text { Barbara Seeger } & \text { NvS: } & \text { Nadja von Samson } \\ \text { CA: } & \text { Christine Axmann } & \text { PO: } & \text { Petra Oberhauser } \\ \text { CS: } & \text { Carola Sedlacek } & \text { SM: } & \text { Sabine Matthes } \\ \text { DF: } & \text { Dieter Fuchs } & \text { SS: } & \text { Siegfried Seifert } \\ \text { DH: } & \text { David M. Hoffmann } & \text { WH: } & \text { Wolfram Huschke } \\ \text { DO: } & \text { Diana Otterbach } & \text { WM: } & \text { Wenka von Mikulicz } \\ \text { FT: } & \text { Felix Tebbe } & \text { WS: } & \text { Walter Salmen } \\ \text { GBS: } & \text { Gabriele Busch-Salmen } & \text { WST: } & \text { Walter Steiner } \\ \text { GG: } & \text { Gerhard Gönner } & \text { WT: } & \text { Wolfgang Timpel } \\ \text { GGU: } & \text { Gitta Günther } & & \end{array}$

\section{Siglenliste}

$\begin{array}{llll}\text { ALH: } & \text { Ausgabe letzter Hand } & \text { Lj: } & \text { Wilhelm Meisters Lehriahre } \\ \text { CiFr: } & \text { Campagne in Frankreich } & \text { MuR: } & \text { Maximen und Reflexionen } \\ \text { DuW: } & \text { Dichtung und Wahrheit } & \text { Tb: } & \text { Tagebücher } \\ \text { Fl: } & \text { Farbenlehre } & \text { Tuj: } & \text { Tag- und Jahreshefte } \\ \text { Gjb: } & \text { Goethe-Jahrbuch } & \text { WA: } & \text { Weimarer Ausgabe } \\ \text { HuD: } & \text { Hermann und Dorothea } & \text { Wj: } & \text { Wilhelm Meisters Wanderjahre } \\ \text { KuA: } & \text { Kunst und Altertum } & & \end{array}$

